\title{
Atividade antimicrobiana de sistemas adesivos contra Streptococcus Mutans, Candida SPP e Staphylococcus Aureus
}

\author{
Antimicrobial activity of adhesive systems against \\ Streptococcus Mutans, Candida SPP and Staphylococcus Aureus
}

\author{
Cecilia Baima Licio' \\ Jessica Castro Teixeira' \\ Márcia Maria de Negreiros Pinto Rocha' \\ Paula Borges Jacques'
}

\begin{abstract}
RESUMO
Objetivo: Analisar o potencial de inibição antimicrobiana de sistemas adesivos dentinários fotoativados, por meio do método da impregnação em papel-filtro esterilizado.

Materiais e métodos: Pesquisa experimental realizada em uma universidade privada na cidade de Fortaleza, em 2015, analisou os sistemas adesivos: Adper Single Bond ${ }^{\mathrm{TM}} 2$ (3M ESPE), Ambar® (FGM), Stae $®$ (SDI), Tetric N-Bond $®$ (Ivoclar Vivadent), Prime \& Bond 2.1® (Dentsply) contra os microrganismos: Streptococcus mutans, Candida albicans e Staphylococcus aureus. Utilizaram-se 63 discos de papel filtro estéreis impregnados, em triplicata, sendo 45 testados para os sistemas adesivos fotoativados, nove para controle positivo com clorexidina $0,12 \%$ e nove para controle negativo com soro fisiológico. Houve semeadura dos três microrganismos em Placas de Petri contendo meio de cultura Ágar Mueller-Hinton e os discos com os adesivos, crescendo por 72 horas a $37^{\circ} \mathrm{C}$ em estufa bacteriológica.

Resultados: Os cinco sistemas adesivos utilizados não apresentaram halo de inibição para Streptococcus mutans. Para Staphylococcus aureus, Ambar ${ }^{\circledR}$ e Stae ${ }^{\circledR}$ apresentaram halo de inibição. Ambar e Adper Single Bond ${ }^{\mathrm{T}} 2 \circledast$ apresentaram halos contra Candida albicans. Os adesivos Tetric N-Bond® e Prime \& Bond $2.1 \circledR$ não apresentaram halo de inibição para os microrganismos testados.

Conclusão: Conclui-se que nenhum dos cinco sistemas adesivos avaliados neste estudo apresentou potencial de inibição antimicrobiana contra Streptococcus mutans, entretanto, houve contra Staphylococcus aureus e Candida spp. Dois dos sistemas não mostraram potencial contra nenhum microrganismo testado. Palavras-chave: Adesivos Dentinários; Candida; Produtos com Ação Antimicrobiana; Staphylococcus aureus; Streptococcus mutans; Testes de Sensibilidade a Antimicrobianos por Disco-Difusão.
\end{abstract}

\begin{abstract}
Purpose: To analyze the antimicrobial inhibition potential of photoactivated dentin adhesives systems through the impregnation method in sterile filter paper.

Materials and methods: Experimental research carried out in a private university in the city of Fortaleza, among the year of 2015, that analyzed the following adhesives systems: Single Bond TM 2 (3M ESPE), Ambar ${ }^{\circledR}(F G M)$, Stae ${ }^{\circledR}$ (SDI), Tetric N-BOND® (Ivoclar Vivadent), Prime \& Bond $2.1 \circledR$ (Dentsply), against the following microorganisms: Streptococcus mutans, Candida albicans, Staphylococcus aureus. It was used 63 impregnated sterile filter paper discs in triplicate, 45 being tested for the photocured adhesive systems, 9 for positive control with chlorhexidine $0.12 \%$ and 9 for negative control with saline solution. There was sowing at the three microorganisms petri dishes containing Mueller-Hinton Ágar culture and the discs with adhesive systems, growing for 72 hours at $37^{\circ} \mathrm{C}$ in a bacteriological incubator.

Results: The five adhesive systems used showed no inhibition zone for Streptococcus mutans. For Staphylococcus aureus, Ambar $\AA$ and Stae $₫$ adhesives systems showed inhibition zone. Ambar $®$ and Single Bond ${ }^{\mathrm{TM}} 2 \circledast$ showed inhibition halos against Candida albicans. Adhesives Tetric N-Bond $\circledast$ and Prime \& Bond $2.1 \circledR$ showed no inhibition zone for the tested microorganisms.

Conclusion: We conclude that none of the five adhesive systems evaluated in this study has the potential of antimicrobial inhibition against Streptococcus mutans, however, there was potential against Staphylococcus aureus and Candida spp. Two of the systems did not show potential against any organism tested.

Key-words: Candida; Dentin-Bonding Agents; Disk Diffusion Antimicrobial Tests; Products with Antimicrobial Action; Staphylococcus aureus; Streptococcus mutans.
\end{abstract}

'Universidade de Fortaleza (UNIFOR), Fortaleza/CE - Brasil 


\section{INTRODUÇÃO}

Os sistemas adesivos são misturas de monômeros hidrofílicos e hidrofóbicos com a capacidade de ligar a resina (porção hidrofóbica) com a dentina (porção hidrofílica), ${ }^{1}$ sendo responsáveis pela união dos materiais restauradores à estrutura dentária. A manutenção e a obtenção de uma adequada união entre o tecido dentário e o material restaurador são essenciais para que haja sucesso dos tratamentos dentários conservadores. ${ }^{2}$

A cavidade oral abriga uma grande variedade de microrganismos, sendo Streptococcus mutans e Candida ssp associadas ao desenvolvimento da cárie dentária. ${ }^{3-5}$ As bactérias Streptococcus mutans são comumente encontradas no biofilme formado às margens das restaurações, principalmente quando o material é a resina composta, fornecendo assim um maior potencial para a instalação de cárie secundária. ${ }^{6}$

Levando em consideração a existência dessas bactérias na superfície dentária, observa-se que o ácido condicionador deveria propiciar atividade antimicrobiana e fortalecer o selamento marginal, visto que a infecção bacteriana é uma das causas principais de resposta inflamatória pulpar associada à restauração. ${ }^{7}$ No entanto, bactérias podem permanecer no interior dos túbulos dentinários após a completa remoção de tecido cariado, evidenciando que o aspecto clínico de tecido dentinário sadio não condiz aos achados microbiológicos. ${ }^{8,9} \mathrm{O}$ condicionamento prévio da dentina com ácido fosfórico quando são utilizados adesivos dentinários convencionais, apesar de eficaz na redução do número de bactérias residuais, não é capaz de eliminá-las em sua totalidade. ${ }^{10-12}$

Os sistemas adesivos podem ser classificados quanto ao número de etapas operatórias, subdividindo-se em três, dois e um passo, dependendo de como essas etapas do condicionamento ácido e hibridização sejam realizadas. Assim, os adesivos da atualidade baseiam-se nas abordagens convencional ou etch-and-rinse (ER) e autocondicionante ou self-etch (SE) (ou etch-and-dry), diferenciando consideravelmente a técnica de adesão relativamente à forma como os adesivos interagem com os tecidos dentários. ${ }^{13}$ Por conseguinte, os adesivos da atualidade são frequentemente conotados como sendo tecnicamente sensíveis, sendo que uma simples falha no procedimento de aplicação clínica pode resultar em uma rápida degradação da interface adesiva e aparecimento precoce de fendas marginais nas restaurações adesivas. ${ }^{13}$

Ainda na atualidade, a longevidade da união dos materiais representa o principal problema quando se estuda a adesão de sistemas adesivos, a qual depende diretamente da capacidade de selamento marginal dos mesmos, sendo fundamental para evitar-se a infiltração marginal, que pode gerar a formação de cáries secundárias. ${ }^{2}$ Essas lesões de cárie são geradas pelo mesmo agente da lesão primária, os quais são capazes de colonizar a interface dente/restauração. Ocorrem de forma similar, tanto quando há colonização na superfície, como lesão primária, ou quando ocorrem através da parede da cavidade, quando há falha no selamento marginal da restauração. ${ }^{14}$

O desenvolvimento de lesões cariosas secundárias ainda é um dos principais motivos para a troca de restaurações, e a alternativa de evitar ou mesmo retardar esse tipo de lesão pode diminuir a necessidade de sua substituição. ${ }^{15}$ Evidencia-se, assim, a necessidade de se utilizar materiais restauradores que apresentem ação antimicrobiana, por exemplo, os sistemas adesivos.

Portanto, a proposição do presente estudo é analisar o potencial de inibição antimicrobiana de sistemas adesivos dentinários fotoativados, por meio do método da impregnação em papel-filtro esterilizado. 


\section{MÉtodos}

Pesquisa experimental realizada no Laboratório de Microbiologia de uma universidade privada em Fortaleza/CE, no período de março a agosto de 2015, que consistiu em um estudo de avaliação comparativa, in vitro, da atividade antimicrobiana de cinco sistemas adesivos dentinários comercializados no mercado brasileiro utilizando-se cepas puras de microrganismos.

Analisaram-se os seguintes sistemas adesivos: Adper Single Bond ${ }^{\mathrm{T}} 2 \AA(3 \mathrm{M}$ ESPE), Ambar® (FGM), Stae ${ }^{\circledR}$ (SDI), Prime \& Bond $2.1 \AA$ (Dentsply), Tetric N-Bond $\AA$ (Ivoclar Vivadent). Os autores afirmam que todos os materiais testados estavam dentro do prazo de validade, sendo avaliados imediatamente após a abertura de seus lacres, e foram selecionados por serem de fácil acesso no mercado odontológico da cidade de Fortaleza/CE.

Todos os sistemas adesivos citados sofreram análise de sua atividade antimicrobiana contra os microrganismos: Streptococcus mutans (ATCC 25175), Candida albicans (ATCC 10231), Staphylococcus aureus (ATCC 6535).

Para a realização dos experimentos, as examinadoras estavam devidamente treinadas e fizeram uso de EPIs (equipamentos de proteção individual), bem como a bancada laboratorial utilizada para a pesquisa estava descontaminada com álcool $70 \%$. Ressalta-se o uso de materiais estéreis para a manipulação dos discos de filtro estéreis e luvas estéreis para a manipulação das placas e dos sistemas adesivos.

Inicialmente, os microrganismos Streptococcus mutans, Candida albicans e Staphylococcus aureus foram inoculados e mantidos por 24 horas em MHB (Mueller-Hinton Broth). Após o crescimento, foram ressuspensos em solução fisiológica a $0,9 \%$ (p.v) até a obtenção de turvação equivalente ao padrão 0,5 da escala de Mc Farland, que corresponde aproximadamente a $1,5 \times 10^{8} \mathrm{UFC} / \mathrm{mL}$. A suspen- são foi semeada em placas de Petri contendo $15 \mathrm{~mL}$ do meio Mueller-Hinton ágar, com uma espessura de aproximadamente $4 \mathrm{~mm}$, com o auxílio de alça bacteriológica, ${ }^{16}$ sempre próximo ao Bico de Bunsen visando a manutenção de condições estéreis.

O teste de inibição do crescimento microbiológico deu-se por ágar-difusão, o qual ocorreu por meio da verificação da formação de halos de inibição de crescimento contornando discos de papel-filtro ${ }^{16}$ contendo os sistemas adesivos, e para isso, utilizaram-se 63 discos de papel-filtro esterilizados no total.

Realizou-se o experimento em triplicata para cada microrganismo (7 discos em cada placa $\times 3=21$ discos $\times 3$ microrganismos testados $=63$ discos) e, desse total, 45 discos de papel-filtro foram utilizados da seguinte forma: 1 para cada sistema adesivo, 9 para controle positivo com clorexidina $0,12 \%$ (manipulada por laboratório Magistral) e 9 para controle negativo com solução de cloreto de sódio 0,9\% (Kabipac - Fresenius Kabi Brasil). Os discos de papel-filtro foram organizados e numerados em placas de Petri de acordo com os sistemas adesivos e os microrganismos.

Os sistemas adesivos foram retirados de seus frascos imediatamente após a remoção do lacre, com o auxílio de uma pipeta manual (Labmate) com ponteiras descartáveis, trocadas a cada sistema adesivo, com o equivalente a uma gota, o que demonstrou ser suficiente para embeber completamente o papel-filtro. Os discos eram então fotoativados a uma distância padronizada de $8 \mathrm{~mm}$, com o fotopolimerizador LED Poly Wireless (KaVo), de acordo com o tempo determinado por seus fabricantes, contido na bula de cada produto.

Os discos com os sistemas adesivos foram colocados nas placas e colocados para o crescimento por $72 \mathrm{~h}$ a $37^{\circ} \mathrm{C}$ em estufa bacteriológica (Biomatic) em aerobiose para Candida albicans e Staphylococcus aureus e em microaerofilia para Streptococcus mutans. 
Após 24 horas, realizou-se a primeira identificação da inibição antimicrobiana dos sistemas adesivos testados. Para a segurança dos resultados, as placas foram mantidas nas mesmas condições por até 72 horas, e então realizada uma nova verificação e confirmação dos resultados.

Os halos de inibição do crescimento dos microrganismos tiveram seus diâmetros aferidos por meio do auxílio de uma régua plástica flexível de 30 centímetros, por duas examinadoras treinadas, nos momentos 24,48 e 72 horas. Realizou-se a média para a identificação do maior e do menor halo de inibição, identificando assim, os adesivos que possuem atividade antimicrobiana e o comportamento das substâncias controle (clorexidina 0,12\% e cloreto de sódio $0,9 \%$ ).

\section{Resultados}

Os cinco sistemas adesivos utilizados na atual pesquisa não apresentaram halo de inibição para a cepa de Streptococcus mutans, o mesmo também verificado no controle com a solução fisiológica $0,9 \%$.

Para confirmar a correta execução do procedimento, a clorexidina $0,12 \%$ (controle positivo) apresentou halo de inibição de 21 $\mathrm{mm}$ nas três placas compostas por Streptococcus mutans.

Nas três placas semeadas com Staphylococcus aureus, dois sistemas adesivos apresentaram halo de inibição neste estudo, Ambar $®$ (FGM) e Stae $®$ (SDI), sendo o Ambarß (FGM) com média das três aferições dos halos de $17,3 \mathrm{~mm}$ e o Stae ${ }^{\circledR}$ (SDI) com uma média de $7 \mathrm{~mm}$ de halo de inibição.

Tal resultado foi confirmado pelo controle positivo com clorexidina $0,12 \%$ que apresentou nas três placas um halo de $12 \mathrm{~mm}$ contra Staphylococcus aureus.

Demais sistemas adesivos e o controle com a solução fisiológica $0,9 \%$ não apresenta- ram inibição do crescimento bacteriano contra Staphylococcus aureus.

O fungo Candida albicans demonstrou sensibilidade, por meio da formação do halo, para dois sistemas adesivos, Ambar (FGM) e Adper Single Bond ${ }^{\mathrm{Tm}} 2 \circledR(3 \mathrm{M}$ ESPE). O sistema Ambar® (FGM) apresentou média de $23 \mathrm{~mm}$ cada e o Adper Single Bond ${ }^{\mathrm{T}} 2 \circledR(3 \mathrm{M}$ ESPE) mostrou de $30 \mathrm{~mm}$.

Para confirmar o procedimento contra a Candida albicans, a clorexidina $0,12 \%$ (controle positivo) evidenciou halo de $11 \mathrm{~mm}$, e os demais sistemas adesivos e a solução fisiológica a $0,9 \%$ não mostraram evidência de inibição de crescimento contra esse fungo.

Os sistemas adesivos Tetric N-Bond $(\mathbb{R}$ (Ivoclar Vivadent) e o Prime \& Bond 2.1® (Dentsply) não apresentaram halo de inibição para os microrganismos testados. É importante ressaltar que as aferições não mudaram em 24, 48 e 72 horas.

A Tabela I apresenta as informações citadas.

\section{Dıscussão}

Quando os pacientes necessitam de tratamento restaurador, inicialmente realiza-se a adequação do meio bucal e é reduzida, consequentemente, a atividade de cárie do paciente, para que, em seguida, sejam feitas as restaurações definitivas e se diminua assim o risco de desenvolvimento de cáries secundárias. ${ }^{17}$ Com a existência de cárie secundária após a realização de tratamento restaurador, há um aumento de interesse na capacidade terapêutica dos materiais dentários restauradores, ${ }^{18}$ dessa forma, os atuais materiais tendem a tornar-se biocompatíveis e bioativos, ou seja, participando ativamente da ação antibacteriana, propriedade com grande importância no controle da cárie dentária. ${ }^{19}$

Uma observação importante é que materiais resinosos e superfícies dos sistemas ade- 
Tabela I - Descrição do resultado acerca da sensibilidade aos materiais testados (média dos halos em mm) e substâncias controle, de acordo com os microrganismos. Fortaleza-CE, 2015.

\begin{tabular}{|c|c|c|c|}
\hline $\begin{array}{l}\text { Microrganismo } \\
\text { Material }\end{array}$ & $\begin{array}{l}\text { Streptococcus } \\
\text { mutans }\end{array}$ & $\begin{array}{l}\text { Candida } \\
\text { albicans }\end{array}$ & $\begin{array}{l}\text { Staphylococcus } \\
\text { aureus }\end{array}$ \\
\hline Adper Single Bond $®$ & Não houve halo & $30 \mathrm{~mm}$ & Não houve halo \\
\hline Ambar® & Não houve halo & $23 \mathrm{~mm}$ & $17,3 \mathrm{~mm}$ \\
\hline Stae ${ }^{\circledR}$ & Não houve halo & Não houve halo & $7 \mathrm{~mm}$ \\
\hline Prime \& Bond $2.1 \circledR$ & Não houve halo & Não houve halo & Não houve halo \\
\hline Tetric N-Bond® & Não houve halo & Não houve halo & Não houve halo \\
\hline $\begin{array}{l}\text { Controle clorexidina } \\
012 \%\end{array}$ & $21 \mathrm{~mm}$ & $11 \mathrm{~mm}$ & $12 \mathrm{~mm}$ \\
\hline $\begin{array}{l}\text { Controle solução } \\
\text { fisiolóqica } 0,9 \%\end{array}$ & Não houve halo & Não houve halo & Não houve halo \\
\hline
\end{tabular}

sivos são mais colonizados por biofilmes orais se comparado a superfícies de outros materiais restauradores (como amálgama, cimento de ionômero de vidro e cerâmica), portanto, a redução da formação de biofilme nas superfícies de materiais à base de resina é considerada um alvo importante para reduzir o risco de cárie secundária e melhorar a longevidade das restaurações. ${ }^{20}$

$\mathrm{Na}$ atual pesquisa, foi analisada a atividade antimicrobiana de sistemas adesivos dentinários contra Candida albicans, Staphylococcus aureus e Streptococcus mutans, por estes serem patógenos diretamente relacionados a doenças na cavidade oral, e quando em desequilíbrio podem ocasionar infecções orais e até mesmo sistêmicas. ${ }^{3-5,6}$

O gênero Candida também pode estar associado à presença de cárie devido às propriedades acidogênicas e capacidade de degradação do colágeno estrutural do tecido dentinário. ${ }^{21}$

$\mathrm{Na}$ presente pesquisa os sistemas adesivos Adper Single Bond ${ }^{\text {TM }} 2 \AA$ e Ambar $®$ apresentaram halos de $23 \mathrm{~mm}$ e $30 \mathrm{~mm}$ respecti- vamente para a Candida ssp, trazendo à tona um resultado satisfatório, já que essa levedura apresenta uma coagregação com Streptococcus mutans, ${ }^{21}$ uma vez que a Candida ssp vem sendo apontada como uma importante coadjuvante no processo da cárie. ${ }^{22}$

Embora a cavidade oral não seja seu habitat normal, o Staphylococcus aureus tem sido isolado a partir de placa dental, particularmente de pacientes com infecção respiratória. É comprovado que dadas as condições orais adequadas, o Staphylococcus aureus pode constituir de fato parte da microbiota oral. ${ }^{23}$

Os resultados deste estudo demonstraram que os sistemas adesivos Ambar ${ }^{\circledR}$ e Stae ${ }^{\circledR}$ apresentaram uma média dos halos de inibição de 17,3 mm e $7 \mathrm{~mm}$, respectivamente, contra Staphylococcus aureus.

O Streptococcus mutans é considerado como um dos microrganismos mais importantes responsáveis pela cárie primária e secundária. ${ }^{24}$

Dados desta pesquisa não foram satisfatórios para Streptococcus mutans, já que nenhum dos cinco sistemas adesivos testa- 
dos apresentou potencial antimicrobiano sobre essa bactéria.

O Streptococcus mutans pode atuar na superfície de diferentes sistemas adesivos, formando biofilme. ${ }^{25}$ Resultados presentes na literatura sugerem que as variações na estrutura química dos componentes presentes nos adesivos ou as técnicas de aplicação podem influenciar na formação do biofilme dentário, mesmo quando um adesivo não contempla em sua composição qualquer formulação antibacteriana específica. ${ }^{19,26}$

O resultado do presente estudo concorda com os resultados encontrados por alguns pesquisadores, que observaram os monômeros Bis-GMA, UDMA e HEMA, monômeros encontrados na formulação química dos cinco sistemas adesivos testados. Esses monômeros apresentaram a possibilidade de adesão e crescimento microbiano, podendo então influenciar na não formação de halos de inibição contra Streptococcus mutans, ${ }^{27}$ portanto, supõe-se que não houve atividade antimicrobiana pelo motivo supracitado.

Os adesivos dentinários testados na atual pesquisa foram avaliados após a fotoativação determinada pelo fabricante, pois os materiais, quando não fotoativados, apresentam uma maior atividade antimicrobiana, e uma metodologia realizada sem fotoativação não representaria adequadamente o processo clínico restaurador. ${ }^{28}$

Assim, os halos de inibição formados pelos sistemas adesivos Adper Single Bond ${ }^{\text {TM }} 2 ®$ (3M ESPE), Ambar ${ }^{\circledR}(F G M)$, Stae ${ }^{\circledR}(S D I)$, quando fotoativados, são explicados pelo fato de não ter ocorrido uma completa conversão de monômeros em polímeros e os monômeros residuais puderam, então, ser liberados para o meio. ${ }^{29}$

Somado a isto, a luz pode não ser capaz de atravessar totalmente o disco de papel-filtro impregnado pelos sistemas adesivos, o que impede que grande parte dos monômeros forme cadeias poliméricas. ${ }^{28}$
Os termos do presente estudo centram-se nos limites de pesquisas in vitro, cujos resultados não podem ser extrapolados para a prática clínica, não significando que os materiais testados não devam ser utilizados. Entretanto, ressalta-se a importância deste trabalho, a fim de dar continuidade às buscas sobre a avaliação antimicrobiana em sistemas adesivos dentinários, principalmente os convencionais, sempre com o intuito de aumentar os índices de sucesso dos tratamentos restauradores. Apesar do estágio de evolução atual das pesquisas com sistemas adesivos, são necessários mais estudos para suprir as limitações existentes (infiltração, dor pós operatória, resistência de união, longevidade de restaurações e potencial de inibição de crescimento de microrganismos).

\section{Conclusão}

Conclui-se que nenhum dos cinco sistemas adesivos fotoativados avaliados neste estudo mostrou potencial de inibição microbiana contra Streptococcus mutans, entretanto, Ambar ${ }^{\circledR}$ e Stae $®$ apresentaram potencial de inibição contra Staphylococcus aureus e Adper Single Bond ${ }^{\mathrm{T}} 2 \circledast$ e Ambar $\circledast$ apresentaram potencial de inibição contra Candida albicans.

Os sistemas adesivos Tetric N-Bond $($ e o Prime \& Bond $2.1 \circledR$ não mostraram potencial contra nenhum microrganismo testado.

\section{REFERÊNCIAS}

1. Brambilla E., et al. Hydrophilicity of dentin bonding systems influences in vitro Streptococcus mutans biofilm formation. Dent Mat, 2014, 30(8):926-35. doi: 10.1016/j. dental.2014.05.009.

1. Coelho A., Canta JP, Martins JNR, Oliveira SA, Marques P. Perspectiva histórica e conceitos atuais dos sistemas adesivos amelodentinários - revisão da literatura. Rev Port Estomatol Med Dent e Cir Maxilofac, 2012, 53:39-46. 
2. Brown LR, Billings RJ, Kaster AG. Quantitative comparisons of potentially cariogenic microorganisms cultured from noncarious and carious root and coronal tooth surfaces. Infect Immun, 1986, 51(3):765-70.

3. Hodson JJ, Craig GT. The Incidence of Candida albicans in the Plaques of Teeth of Children. Dent Practit, 1972, 22(8):296-301.

4. Kaminishi H., Hagihara Y., Hayashi S, Cho T. Isolation and characteristics of collagenolytic enzyme produced by Candida albicans. Infect Immun, 1986, 53(2):312-16.

5. Svanberg M., Mjör IA, Orstavik D. Mutans streptococci in plaque from margins of amalgam, composite, and glass-ionomer restorations. J Dent Res, 1990, 69(3):861-4.

6. Pashley DH, Carvalho R. Dentin permeability and adhesion. J Dent, 1997, 25(5):355-372.

7. Kidd EA, Joyston-Bechal S., Beighton D. Microbiological validation of assessments of caries activity during cavity preparation. Caries Res, 1993, 27(5):402-8.

8. Kidd EA, Joyston-Bechal S., Beighton D. The use of a caries detector dye during cavity preparation: a microbiological assessment. Br Dent J, 1993, 174(7):245-8.

9. Jensen OE, Handelman SL. Effect of an autopolymerizing sealant on viability of microflora in occlusal dental caries. Scand J Dent Res, 1980, 88(5):382-8.

10. Kramer PF, Zelante F., Simionato MR. The immediate and long-term effects of invasive and noninvasive pit and fissure sealing techniques on the microflora in occlusal fissures of human teeth. Pediatr Dent, 1993, 15(2):108-12.

11. Luglie PF, Delitala PP, Zanetti S., Sanna S. An in-vivo bacteriological study on the effects of acid etching at the bottom of cavities. Minerva Stomatol, 1998, 47(1-2):19-26.

12. Meerbeek BV, Yoshihara K., Yoshida Y., Mine A., Munck J., Landuyt KV. State of the art of self-etch adhesives. Dent Mater, 2011, 27:1728. doi: 10.1016/j.dental.2010.10.023.

13. Tantbirojn D., Douglas, WH, Versluis A. Inhibitive effect of resin-modified glass ionomer cement on remote enamel artificial caries. Caries Res, 1997, 31(4):275-80.
14. Fontana M., Zero DT. Assessing patients' caries risk. J Am Dent Assoc, 2006, 137:1231-9.

15. Oplustil CP, Zoccoli CM, Tobouti NR, Sinto SI. Procedimentos básicos em microbiologia clínica, 2. ed., São Paulo: Sarvier, 2004.

16. Perito, MAM, Jorge, ACT, Cassonic, A., Rodrigues JA. Uso do laser na prevenção da cárie dental. Rev Dent On Line, 2009, 8(18):35-40.

17. Esteves CM, Reis AF, Rodrigues JA. Atividade antibacteriana de sistemas adesivos autocondicionantes. Rev Saude UNG, 2010, 4(1):10-7.

18. Imazato, S. Bio-active restorative materials with antibacterial effects: new dimension of innovation in restorative dentistry. Dent Mater J, 2009, 28:11-9.

19. Zhang K., Melo MA, Cheng L., Weir MD, Bai Y., $\mathrm{Xu} \mathrm{HH}$. Effect of quaternary ammonium and silver nanoparticle-containing adhesives on dentin bond strength and dental plaque microcosm biofilms. Dent Mater, 2012, 28:842-52. doi: 10.1016/j.dental.2012.04.027.

20. Metwalli KH, Khan SA, Krom BP, Jabra-Rizk MA. Streptococcus mutans, Candida albicans, and the Human Mouth: A Sticky Situation.

PLoS Pathog, 2013, 9(10). doi:10.1371/journal.ppat.1003616.

21. Charone S., Portela MB, Chagas MS, de Araújo Soares RM, Castro GF. Biofilm of Candida albicans from oral cavity of an HIV infected child: challenge on enamel microhardness. Oral Surg Oral Med Oral Pathol Oral Ra-diol, 2013, 115(4):500-4. doi: 10.1016/j.000o.2012.11.003.

22. Thurnheer, T., Belibasakis, GN. Integration of non-oral bacteria into in vitro oral biofilms. Virulence, 2015, 6(3):258-64.

23. Kutsch VK, Young DA. New directions in the etiology of dental caries disease. J Calif Dent Assoc, 2011, 39:716-21.

24. Pinheiro SL, Soares HH, Ribeiro MC. Microbial contamination and inhibitory effect against Streptococcus mutans from fifth-generation bonding systems. J Appl Biomater Biomech, 2010, 8:52-5.

25. Paradella TC, Koga-Ito CY, Jorge AO. In vitro antibacterialactivity of adhe-sive systems on Streptococcus mutans. J Adhes Dent, 2009,1 1:95-9. 
26. Brambilla E., Cagetti MG, Cagliani M., Fadini L., Godoy FG, Strohmenger L. Influence of different adhesive restorative materials on mutans streptococci colonization. Am J Dent, 2005, 18:173-6.

27. Imazato S., Kuramoto A., Kaneko T., Ebisu S., Russell RR. Comparison of antibacterial activity of simplified adhesive systems. Am J Dent, 2002, 15(6):356-360.
28. Cadenaro M., Antoniolli F., Sauro S., Tay FR, Di Lenarda R., Prati C., et al. Degree of conversion and permeability of dental adhesives. Eur $\mathbf{J}$ Oral Sci, 2005, 113(6):525-30.

Este manuscrito não apresenta conflitos de interesse.

Submetido em: 28-6-2016

Aceito em: 23-8-2016 